\title{
Successful management of reversible cerebral vasoconstriction syndrome-induced intracerebral hemorrhage with minimally invasive surgery plus recombinant tissue-type plasminogen activator
}

\begin{abstract}
Background: Reversible cerebral vasoconstriction syndrome (RCVS) is characterized by thunderclap headaches associated with radiographic evidence of reversible segmental vasoconstriction of cerebral arteries.RCVS is associated with both ischemic and hemorrhagic strokes.

Methods: We present a case of probable RCVS with intracerebral hemorrhage (ICH) in the setting of antidepressant medication use that was successfully managed with minimally invasive surgery plus intra-hematomal delivery of recombinant tissue plasminogen activator (rtPA)

Results: A 45-year-old woman underwent an elective repair of a left superior semicircular canal dehiscence. On postoperative day 3 she developed a severe headache followed by acute onset of left face and body hemiparesis. Head computed tomography (CT) showed a large acute ICH in the right basal ganglia. Due to concern for impending cerebral herniation, the patient underwent stereotactic aspiration of ICH and placement of an indwelling catheter for intra-hematomal delivery of rtPA. Seven doses of $1 \mathrm{mg}$ rtPA were administered via the intraparechymal catheter with significant clot reduction. Repeat cerebral angiogram showed arterial beading in both the anterior and posterior circulation.

Conclusion: RCVS is an important diagnosis to consider in cases of ICH without identifiable cause even when initial angiography is normal. In severe cases, secondary management of ICH plays an important role, including minimally invasive surgical intervention to reduce clot volume and minimize perihematomal edema.
\end{abstract}

Keywords: Intracerebral hemorrhage, Tissue plasminogen activator, Cerebral edema, Thrombolysis, Minimally invasive surgery
Volume I Issue 5 - 2014

\author{
Lucia Rivera,' Saman Nekoovaght-Tak, ${ }^{2}$ \\ Deanna Saylor,' Judy Huang, 'Victor Urrutia,' \\ Wendy C Ziai' \\ 'Department of Neurology, Johns Hopkins University School of \\ Medicine, USA \\ ${ }^{2}$ Division of Brain Injury Outcomes, Johns Hopkins University \\ School of Medicine, USA \\ ${ }^{3}$ Department of Neurosurgery, Johns Hopkins University School
} of Medicine, USA

\begin{abstract}
Correspondence: Wendy C. Ziai, Department of Neurology, Johns Hopkins University School of Medicine, The Johns Hopkins Hospital, 6 Wolf Street, Baltimore, MD 2I23I, USA, Tel 4I0-614-1646, Fax 410-502-7869, Email weziai@jhmi.edu
\end{abstract}

Received: July 18, 2014 | Published: September 13, 2014
Abbreviations: RCVS, Reversible Cerebral Vasoconstriction Syndrome; rtPA, recombinant tissue Plasminogen Activator; GCS, Glasgow Coma Scale; CT, Computed Tomography; ICH, Intracerebral Hemorrhage; MRI, Magnetic Resonance Imaging; MRR, MR Renography; ACA, Anterior Cerebral Artery; MIS, Minimally Invasive Surgery; TCD, Transcranial Doppler; ICU, Intensive Care Unit; MISTIE, Minimally Invasive Surgery plus rt-PA in the Treatment of Intracerebral hemorrhage; SAH, Subarachnoid Hemorrhage

\section{Introduction}

Reversible cerebral vasoconstriction syndrome (RCVS), also known as Call-Fleming syndrome is characterized by thunderclap headaches associated with radiographic evidence of reversible segmental vasoconstriction of cerebral arteries with subsequent resolution. More than half of cases of RCVS occur postpartum or in association with exposure to serotonergic or adrenergic medications, however, multiple etiologies and underlying mechanisms are likely and many cases have no obvious cause. ${ }^{1}$ RCVS is associated with both ischemic and hemorrhagic strokes. Cortical subarachnoid hemorrhage is the most common pattern, but intracerebral and subdural hemorrhageshave also been described. ${ }^{2-7}$

Here we present a case of probable RCVS in the setting of longterm use of antidepressant medications and recent auditory surgery that was successfully managed with minimally invasive surgery plus intra-hematomal delivery of recombinant tissue plasminogen activator (rtPA).

\section{Case presentation}

A 45 year old woman with a history of migraines and depression, treated with fluoxetine $40 \mathrm{mg}$ daily underwent an elective repair of a left superior semicircular canal dehiscence for symptoms of dizziness, autophony and pulsatile tinnitus. The surgical procedure consisted of a left middle cranial fossa approach and plugging of the superior semicircular canal with titanium cranioplasty. One day prior to surgery the patient reported an acute severe headache different from her usual migraines. The headache was associated with tingling and an uncomfortable sensation in her left arm. She denied use of drugs or substances linked to vasoconstriction. She also reported a severe, but not atypical headache while driving to the hospital. Following an uncomplicated procedure, on postoperative day 3 she developed a severe headache followed by acute change in mental status (Glasgow coma scale (GCS) decreased from 14 to 8 ). Blood pressure at presentation was136/61(compared to baseline 103/69). Neurological exam was remarkable for new left face and body hemiparesis. Head computed tomography (CT) showed an acute intracerebral hemorrhage (ICH) in the right basal ganglia with calculated volume of $21.1 \mathrm{cc}$ (Figure 1). The patient was intubated for airway protection and treated with hyperosmolar therapy. Repeat head CT within 2 hours showed hematoma expansion, ICH volume measuring $39.7 \mathrm{cc}$ 
with worsening edema and increasing midline shift $(7 \mathrm{~mm}$ at septum pellucidum). Four-vessel cerebral angiography performed on day of ICH presentation did not reveal any vascular lesions or vasospasm. Head CT on day 2 showed mild hematoma expansion (volume of $42.8 \mathrm{cc}$ ). Due to concern for impending cerebral herniation with worsening GCS to7; the patient underwent a stereotactic right frontal burrhole placement for percutaneous aspiration of ICH and placement of an indwelling ventricular catheter for delivery of rtPA directly into the ICH for thrombolysis. In the Neuro ICU, seven doses of $1 \mathrm{mg}$ rtPA every 8 hours were administered via the intraparechymal catheter After each dose the catheter was clamped for 1 hour. Between doses, gravity drainage of blood and CSF yielded 50 to $116 \mathrm{cc} /$ day. Following three days of rtPA infusion, head CT showed significant reduction in clot volume to $15.1 \mathrm{cc}$ (Figure 1). The intraparenchymal catheter was removed two days later (five days after placement) to allow further drainage with a final clot volume of $8.2 \mathrm{cc}$. On ICU day 5, the patient was awake and following commands, her GCS was 14 and NIHSS 15. She was extubated and transferred to the neurology stroke service.

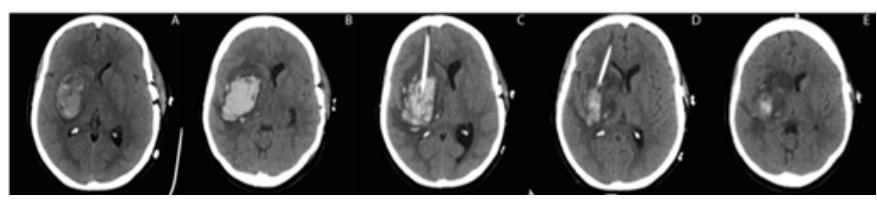

Figure I

A. Non-contrast CT of the head shows an acute intracerebral hemorrhage $(\mathrm{ICH})$ in the right basal ganglia with moderate effacement of right lateral and third ventricles $(\mathrm{ICH}$ volume $=2 \mathrm{I} . \mathrm{Icc})$.

B. CT head on day 2 shows hematoma expansion ( $\mathrm{vol}=42.8 \mathrm{cc}$ ).

C. CT head post stereotactic aspiration of $\mathrm{ICH}$ with indwelling catheter.

D. CT head after seven doses of rtPA delivered directly into hematoma cavity with gravity drainage ( $\mathrm{vol}=\mid 5 . \mathrm{Icc})$.

E. CT head $48 \mathrm{hrs}$ after last dose of rtPA and catheter removal ( $\mathrm{vol}=8.2 \mathrm{cc}$ ).

Magnetic resonance imaging (MRI) at 10 days after $\mathrm{ICH}$ onset showed ischemic changes in the right caudate nucleus and corticospinal tract.MR venography (MRV) was negative for sinus thrombosis and MRA showed no definite vascular malformation. A repeat angiogram performed eleven days after ICH onset, showed mild beaded irregularity of the distal right anterior cerebral artery (ACA), posterior cerebral artery, middle cerebral artery and left ACA branches (Figure 2). In view of these findings consistent with RCVS, verapamil (20mg PO q8hrs) and intravenous magnesium sulfate ( $2 \mathrm{gm}$ IV q4hrs) were administered for five days followed by oral magnesium oxide (400mg PO qday). Magnesium levels were 2 to $3.6 \mathrm{mg} / \mathrm{dl}$. Transcranial Doppler (TCD) at 2 weeks from ICH onset showed mildly elevated CBFV's in the right middle and anterior cerebral arteries and in both internal carotid arteries but without TCD signs of vasospasm.

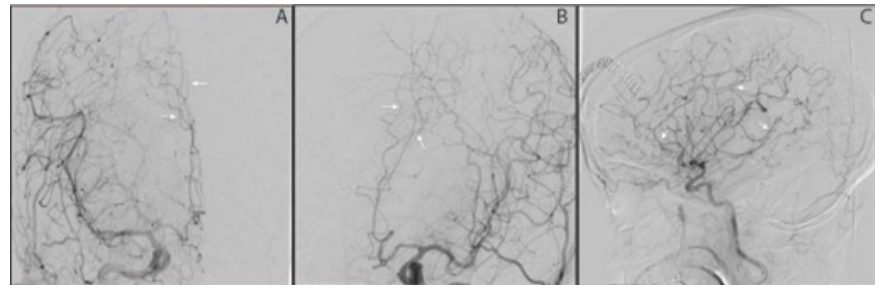

Figure 2 Cerebral angiogram anterior-posterior (AP) view after contrast injection of right $(A)$ and left $(B)$ internal carotid artery (ICA) showing mild beaded irregularity of distal anterior cerebral arterybranches and $(C)$ sagittal view contrast injection of right ICA showing mild beaded irregularity of distal middle cerebral artery branches.
The patient was discharged to acute rehabilitation 19 days after admission at which time NIHSS was 14 . She was alert, talking, oriented, following commands with persistent left homonymous hemianopsia, left hemiplegia and left hemineglect.

At four weeks following hospital discharge left hemiplegia had improved and she was able to walk short distances with a cane. She did not resume any antidepressants and has had no new focal symptoms after 6 months of follow-up (NIHSS 13 at 6 months).

\section{Discussion}

In this case, historical data including young age and lack of premorbid or pre-event hypertension did not reveal any other cause of spontaneous ICH; the diagnosis of RCVS was considered based on history of prolonged use of the selective serotonin reuptake inhibitor fluoxetine.

SSRI exposure is associated with an increased risk of intracerebral bleeding although the data is conflicting. ${ }^{8}$ Serotonin is released by platelets at the site of vascular injury to promote clot formation. Platelets contain a serotonin reuptake transporter that is similar to that in brain. ${ }^{9}$ SSRIs inhibit this transporter inplatelets. ${ }^{2,10}$ In one study, platelet serotonin concentrations decreased by $83 \%$ after 2 weeks of therapeuticparoxetine administration. ${ }^{2}$ In a case-control study of 916 hemorrhagic stroke patients, and 1776 demographically matched controls, after controlling for multiple risk factors, there was no increased risk for $\mathrm{ICH}$ or subarachnoid hemorrhage (SAH). ${ }^{9}$ However, a recent meta-analysis reported a low, but increased risk for $\mathrm{ICH}$, estimated at 1 additional intracerebral bleeding episode per 10,000 persons treated for 1 year. ${ }^{7}$ In addition, SSRI exposure in combination with oral anticoagulants was associated with an increased risk of bleeding compared with oral anticoagulants alone. ${ }^{8}$

The location of this patient's hemorrhage in the deep basal ganglia is consistent with reported cases of RCVS-associated ICH. Ducros et al. ${ }^{4}$ reported 89 consecutive patients with RCVS of whom 11 had $\mathrm{ICH}(12 \%) ; 4$ of these were located in the basal ganglia. Hemorrhagic manifestations of RCVS were more frequent in older patients, females, and migraners (without aura) which all match our patient profile. Patients with hemorrhage were also more likely to have cerebral infarction ( $13 \%$ vs. $2 \%$ without hemorrhage). The ischemic changes detected in our patient may have been due to compressive effects of the ICH although vasospasm cannot be ruled out.

The lack of initial angiographic evidence of cerebral vasoconstriction in this patient at the time of hemorrhage does not rule out RCVS. In Ducros' series, vasoconstriction on neuroimaging was diagnosed after the hemorrhage in $10 / 30$ patients with an initial normal vascular study. ${ }^{4}$ The mean delay from headache onset to a second angiogram showing vasoconstriction was 8.2 days which is similar to our patient. In addition, over half of patients, including patients with hemorrhage have a normal initial brain image at time of headache onset, ${ }^{11}$ as did our case. It is possible that the mild vasospasm detected on repeat angiography in this case was related to procedural manipulation or the hemorrhage itself although it involved vessels clearly remote from the site of bleeding or catheter placement.

Headache triggers are fairly commonly reported in association with RCVS (18/89 in Ducros' series). ${ }^{4}$ Prior reports of cranial surgery associated with symptomatic vasospasm include intracranial tumor resection and carotid surgery. ${ }^{12-14}$ In our case, the temporal association with auditory surgery may be coincidental. The initial complaint of headache occurred preoperatively and the surgery did not involve the intracranial contents. 
Clinical outcomes in patients with hemorrhagic RCVS are notably worse than in patients without hemorrhage as this case clearly demonstrates. Although there is no established treatment for RCVS, most clinicians administer calcium channel blockers and intravenous magnesium, in addition to supportive management for headache control with analgesics, and continuous blood pressure monitoring to maintain normotensive goals. Interventional procedures such as angioplasty have also been reported..$^{15}$ In our case the diagnosis of RCVS was not initially appreciated in view of the unremarkable initial angiogram and the ICH management took precedence due to impending cerebral herniation. The use of minimally invasive surgery (MIS) for spontaneous ICH dates back to the 1960s. Since then and more recently thrombolytic infusion through a stereotactically placed catheter. ${ }^{16-19}$ Use of rtPA in MIS is associated with accelerated clot thrombolysis without increased risk of hemorrhage or other adverse events. ${ }^{20,21}$ The MISTIE II trial, a phase II multicenter study randomized patients into medical treatment $(\mathrm{N}=42)$ vs. MIS, either alone $(\mathrm{N}=10)$ or with intraparenchymal administration of $1 \mathrm{mg}$ rtPA dosed every 8 $\mathrm{h}(\mathrm{N}=69)$. Clotreduction was greater in the surgical group with an average residual, MIS techniques have continued to evolve with the use of ultrasound techniques hematoma volume for surgical patients of 19.6 versus $40.7 \mathrm{ml}$ in the medical group at comparable time points post-intervention. Perihematomaledema was also less in the surgical group and its reduction was positively correlated with reduction in ICH volume. Intra-hematomal thrombolysis did not appear to have any neurotoxic effects. At this time, there is sufficient evidence that in selected cohorts of patients with ICH, stereotactic aspiration and thrombolytic clot removal are feasible and exhibit trends to improved 30-day survival..$^{22}$ Long-term outcomes are under investigation with the currently enrolling MISTIE III trial, a phase III, randomized, multicenter study comparing image-guided catheter-based removal of blood clot with intrahematomal rtPA versus best medical management in subjects with spontaneous ICH volume $>25 \mathrm{cc}$.

The reduction in hematoma size in this patient, from $42.8 \mathrm{cc}$ to $8.2 \mathrm{cc}$ is consistent with the goal of maximizing clot evacuation that is correlated with decreased volume of perihematomal edema, and potentially decreased risk of herniation and better recovery. Maximal clot reduction was facilitated by the following procedures: waiting until the ICH had stabilized (at least 6 hours after expansion); optimal catheter placement along the central longitudinal axis of the clot to maximize exposure of the hematoma to rtPA; daily head CT scans to assess progress and potential bleeding; and continuing catheter drainage for an additional 24-48 hours after the final dose of rtPA which decreased hematoma volume by an additional $7 \mathrm{cc}$ taking advantage of the prolonged half-life of rtPA within a hematoma cavity. We did not detect any toxicity related to rtPA administration in this patient.

\section{Conclusion}

RCVS is an important diagnosis to consider in cases of ICH without identifiable cause even when initial angiography is normal. In severe cases, aggressive management of ICH plays an important role, including minimally invasive surgical intervention to reduce clot volume and minimize perihematomal edema. In this case, we believe that this approach allowed better functional recovery than medical management alone. MIS plus rtPA for ICH evacuation has promising results, but must be practiced within a strict safety protocol to optimize clinical benefit.

\section{Acknowledgments}

None.

\section{Conflicts of interest}

None.

\section{References}

1. Werring DJ. Reversible cerebral vasoconstriction syndrome and intracranial hemorrhage: some answers, many questions. Stroke. 2010;41(11):2455-2456

2. Javors MA, Houston JP, Tekell JL, et al. Reduction of platelet serotonin content in depressed patients treated with either paroxetine or desipramine. Int J Neuropsychopharmacol. 2000;3(3):229-235.

3. Ducros A. Reversible cerebral vasoconstriction syndrome. Lancet Neurol. 2012;11(10):906-917.

4. Ducros A, Fiedler U, Porcher R, et al. Hemorrhagic manifestations of reversible cerebral vasoconstriction syndrome: frequency, features, and risk factors. Stroke. 2010;41(11):2505-2511.

5. Ducros A, Boukobza M, Porcher R, et al. The clinical and radiological spectrum of reversible cerebral vasoconstriction syndrome. A prospective series of 67 patients. Brain. 2007130(pt 2):3091-3101.

6. Chen SP, Fuh JL, Wang SJ, et al. Magnetic resonance angiography in reversible cerebral vasoconstriction syndromes. Ann Neurol. 201067(5):648-656

7. Chen SP, Fuh JL, Lirng JF, et al. Recurrent primary thunderclap headache and benign CNS angiopathy: spectra of the same disorder? Neurology. 2006;67(12):2164-2169.

8. Hackam DG, Mrkobrada M. Selective serotonin reuptake inhibitors and brain hemorrhage: a meta-analysis. Neurology. 2012;79(18):18621865 .

9. Kharofa J, Sekar P, Haverbusch M, et al. Selective serotonin reuptake inhibitors and risk of hemorrhagic stroke. Stroke. 2007;38(11):30493051 .

10. Hatanaka K, Nomura T, Hidaka K, et al. Biochemical profile of YM992, a novel selective serotonin reuptake inhibitor with 5-HT2A receptor antagonistic activity. Neuropharmacology. 1996;35(11):1621-1626.

11. Singhal AB, Hajj-Ali RA, Topcuoglu MA, Fok J, et al. Reversible cerebral vasoconstriction syndromes: analysis of 139 cases. Arch Neurol. 2011;68(8):1005-1012.

12. Hyde-Rowan MD, Roessmann U, Brodkey JS. Vasospasm following transsphenoidal tumor removal associated with the arterial changes of oral contraception. Surg Neurol. 1983;20(2):120-124.

13. LeRoux PD, Haglund MM, Mayberg MR, et al. Symptomatic cerebral vasospasm following tumor resection: report of two cases. Surg Neurol. 1991;36(1):25-31.

14. Schaafsma A, Veen L, Vos JP. Three cases of hyperperfusion syndrome identified by daily transcranial Doppler investigation after carotid surgery. Eur J Vas Endovasc Surg. 2002;23(1):17-22.

15. Ioannidis I, Nasis N, Agianniotaki A, Reversible cerebral vasoconstriction syndrome: treatment with multiple sessions of intra-arterial nimodipine and angioplasty. Interv Neuroradiol. 2012;18(3):297-302.

16. Kandel EI, Peresedov VV. Stereotaxic evacuation of spontaneous intracerebral hematomas. J Neurosurg. 1985;62(2):206-213.

17. Niizuma H, Suzuki J. Computed tomography-guided stereotactic aspiration of posterior fossa hematomas: a supine lateral retromastoid approach. Neurosurgery. 198721(3):422-427.

18. Newell DW, Shah MM, Wilcox R, et al. Minimally invasive evacuation of spontaneous intracerebral hemorrhage using sonothrombolysis. $J$ Neurosurg. 2011115(3):592-601.

Citation: Rivera L, Nekoovaght-Tak S, Saylor D, et al. Successful management of reversible cerebral vasoconstriction syndrome-induced intracerebral hemorrhage with minimally invasive surgery plus recombinant tissue-type plasminogen activator.J Neurol Stroke. 20I4; I (5): I60-163. 
19. Vespa P, McArthur D, Miller C, et al. Frameless stereotactic aspiration and thrombolysis of deep intracerebral hemorrhage is associated with reduction of hemorrhage volume and neurological improvement. Neurocrit Care. 2005;2(3):274-281.

20. Mould WA, Carhuapoma JR, Muschelli J, et al. Minimally invasive surgery plus recombinant tissue-type plasminogen activator for intracerebral hemorrhage evacuation decreases perihematomal edema. Stroke. 2013;44(3):627-634.
21. Carhuapoma JR, Barrett RJ, Keyl PM, et al. Stereotactic aspirationthrombolysis of intracerebral hemorrhage and its impact on perihematoma brain edema. Neurocrit Care. 2008;8(3):322-329.

22. Barrett RJ, Hussain R, Coplin WM, et al. Frameless stereotactic aspiration and thrombolysis of spontaneous intracerebral hemorrhage. Neurocrit Care. 2005;3(3):237-245.

Citation: Rivera L, Nekoovaght-Tak S, Saylor D, et al. Successful management of reversible cerebral vasoconstriction syndrome-induced intracerebral hemorrhage with minimally invasive surgery plus recombinant tissue-type plasminogen activator. J Neurol Stroke. 20 I4; I (5): I60-163. 